\title{
Púrpura trombocitopénica posterior a vacunación contra COVID-19
}

\section{Thrombocytopenic purpura following COVID-19 vaccination}

\author{
Luis Andrés Dulcey-Sarmiento, Raimondo Caltagirone-Micelli • Mérida (Venezuela) \\ Álvaro luis Ruge-Serrano, Manuel David Cantillo-Reines • Barranquilla (Colombia) \\ Pedro Nel Hernández-Anaya, Cristian Ornandy Henao-Niño • Bucaramanga (Colombia)
}

DOI: https://doi.org/10.36104/amc.2022.2268

\section{Resumen}

Introducción: la pandemia por COVID-19 ha generado un costo inexorable a los gobiernos y una mortalidad elevada en términos generales, el proceso de vacunación es la única estrategia efectiva en disminuir la morbimortalidad en general, sin embargo, dicha administración no está exenta de riesgos.

Presentación del caso: presentamos el caso clínico de una paciente de género femenino de la séptima década, quien es vacunada contra el COVID-19 y tras cuatro días posteriores a la segunda dosis de vacuna anti COVID-19, comienza a presentar lesiones purpúricas que inician en miembros inferiores y ascienden progresivamente hasta el tronco asociado a artralgias intensas, dolor abdominal y náuseas. Es ingresada encontrándose recuentos plaquetarios menores a 10 mil plaquetas y se hace el diagnóstico de una púrpura trombocitopénica inmune asociada a la vacuna de AstraZeneca contra COVID-19, iniciándose terapia inmunosupresora específica.

Discusión: el presente caso constituye la aparición de una púrpura trombocitopénica idiopática, patología poco frecuente con un amplio repertorio de eventos desencadenantes encontrándose las infecciones y reacciones vacunales dentro de los principales detonantes. La presente reacción vacunal es poco frecuente siendo lo anecdótico en específico la presencia de púrpura trombocitopénica inmune, hasta el momento son muy escasas las descripciones en la literatura sobre este tipo de reacción vacunal razón por la cual se decide recopilar y publicar el mismo mencionando la evidencia científica disponible más reciente sobre el tema. (Acta Med Colomb 2022; 47. DOI: https://doi.org/10.36104/amc.2022.2268).

Palabras clave: vacuna, púrpura, trombocitopenia, inmunológico.

\section{Abstract}

Introduction: the COVID-19 pandemic has generated an inexorable cost for governments and an elevated mortality overall. Vaccination is the only effective strategy for decreasing overall morbidity and mortality; however, this vaccination is not without risks.

Case presentation: we present the clinical case of a female patient in her seventies who was vaccinated against COVID-19 and four days after the second dose of the COVID-19 vaccine developed purpuric lesions beginning on her lower limbs and ascending progressively towards the trunk, associated with intense arthralgias, abdominal pain and nausea. She was admitted with platelet counts of less than 10,000, was diagnosed with immune thrombocytopenic purpura associated with the AstraZeneca COVID-19 vaccine, and was started on specific immunosuppressive therapy.

Discussion: this is a case of idiopathic thrombocytopenic purpura, a rare pathology with a broad repertoire of triggering events, with infections and vaccine reactions among the main triggers. This vaccine reaction is rare, with the specific anecdotal point being the presence of immune thrombocytopenic purpura. To date, there are very few descriptions of this type of vaccine reaction in the literature, which is why we chose to compile and publish it, mentioning the most recent scientific evidence available on the subject. (Acta Med Colomb 2022; 47. DOI: https://doi.org/10.36104/amc.2022.2268).

Key words: vaccine, purpura, thrombocytopenia, immune.
Dres. Luis Andrés Dulcey-Sarmiento, Raimondo Caltagirone-Micelli: Internistas Universidad de los Andes de Mérida (Venezuela); Dr. Alvaro Luis Ruge-Serrano: Médico General Universidad Metropolitana de Barranquilla; Dr. Manuel David Cantillo-Reines: Médico General Universidad del Magdalena. Barranquilla (Colombia); Dres. Pedro Nel Hernández-Anaya, Cristian Ornandy Henao-Niño: Médico General Universidad Industrial de Santander. Bucaramanga (Colombia).

Correspondencia: Dr. Luis Andrés DulceySarmiento. Mérida (Venezuela).

E-Mail: luismedintcol@gmail.com Recibido: 16/VIII/2021 Aceptado: 15/X/2021 


\section{Introducción}

La pandemia por COVID-19 ha generado una mortalidad elevada en términos generales, el proceso de vacunación es la única estrategia efectiva en disminuir la morbimortalidad, sin embargo, dicha administración no está exenta de riesgos (1-2).

La presencia de trombocitopenia inmunológica relacionada con la vacunación contra el COVID-19 es escasamente frecuente, existen pocos reportes descritos, sus criterios diagnósticos no han sido claramente validados, razón por la cual hemos decidido recopilar el presente caso con el fin de que pueda aportar a reconocer este tipo de evento adverso.

\section{Presentación del caso}

Se trata de una paciente de género femenino de la séptima década de la vida que ingresa, ya que al cuarto día de su segunda dosis de AstraZeneca contra COVID-19, comienza a presentar lesiones purpúricas en miembros inferiores y ascienden progresivamente hasta el tronco asociado a artralgias, dolor abdominal y naúseas, ingresa encontrándose recuentos plaquetarios en 13 000. Dentro de sus antecedentes sólo presenta hipertensión en manejo con losartán 50 $\mathrm{mg} / 2$ diarias. Niega ingesta de medicación antiagregante o anticoagulante. Al examen físico hemodinamia estable, signos vitales dentro de la normalidad, afebril, neurológico sin déficit, tórax sin alteraciones y el abdomen sin visceromegalias o alteraciones. Piel evidencia lesiones purpúricas dispersas en miembros inferiores de carácter ascendente llegando hasta el tronco menores de $5 \mathrm{~mm}$, no desaparecen a la digitopresión (Figuras 1-3).

Se pruebas de hisopado para COVID negativas. Durante la hospitalización se ordenó igualmente anticuerpos antinucleares y Anti-DNA de doble cadena en búsqueda de colagenopatías. Se ordenan durante su seguimiento estudios de química, función renal, hepática y electrolitos, los niveles de dímero D eran mayores de 10 000. A las 48 horas las plaquetas se encontraban en 8000 , a las 72 horas en 5000, no se presentaron eventos de sangrado durante su estancia.

Se midieron niveles de inmunoglobulina dentro de límites normales. Las serologías para virus hepatotropos, herpes, VIH y Epstein Barr negativas. Ante el amplio repertorio de estudios ordenados siendo todos negativos se considera que el evento desencadenante de la trombocitopenia inmune fue la vacunación cuatro días antes del inicio de todo el cuadro clínico. requirió de corticoides sin mejoría, razón por la cual se inició a los cinco días inmunoglobulina $1 \mathrm{~g} /$ kilo/día por tres dosis con 27000 plaquetas a partir de las $48 \mathrm{~h}$ de iniciada la terapia. Transcurridos ocho días de hospitalización los recuentos plaquetarios posteriores se encuentran por encima de 50000 plaquetas para posteriormente dar de alta a los 13 días con 11400 plaquetas para continuar manejo por hematología.

\section{Discusión}

El presente caso es anecdótico dado la escasa frecuencia de trombocitopenia inducida tras la vacunación para COVID-19. La Sociedad Alemana de Trombosis y Hemostasia descubrió que de aproximadamente 2.2 millones de dosis de vacuna AstraZeneca administradas hasta el momento de la publicación de su artículo, se habían reportado un total de 31 casos de trombosis, estas ocurrieron 4-16 días después y se notificó trombocitopenia en 19 pacientes, con un curso clínico fatal en nueve (3). En este tipo de eventos también se
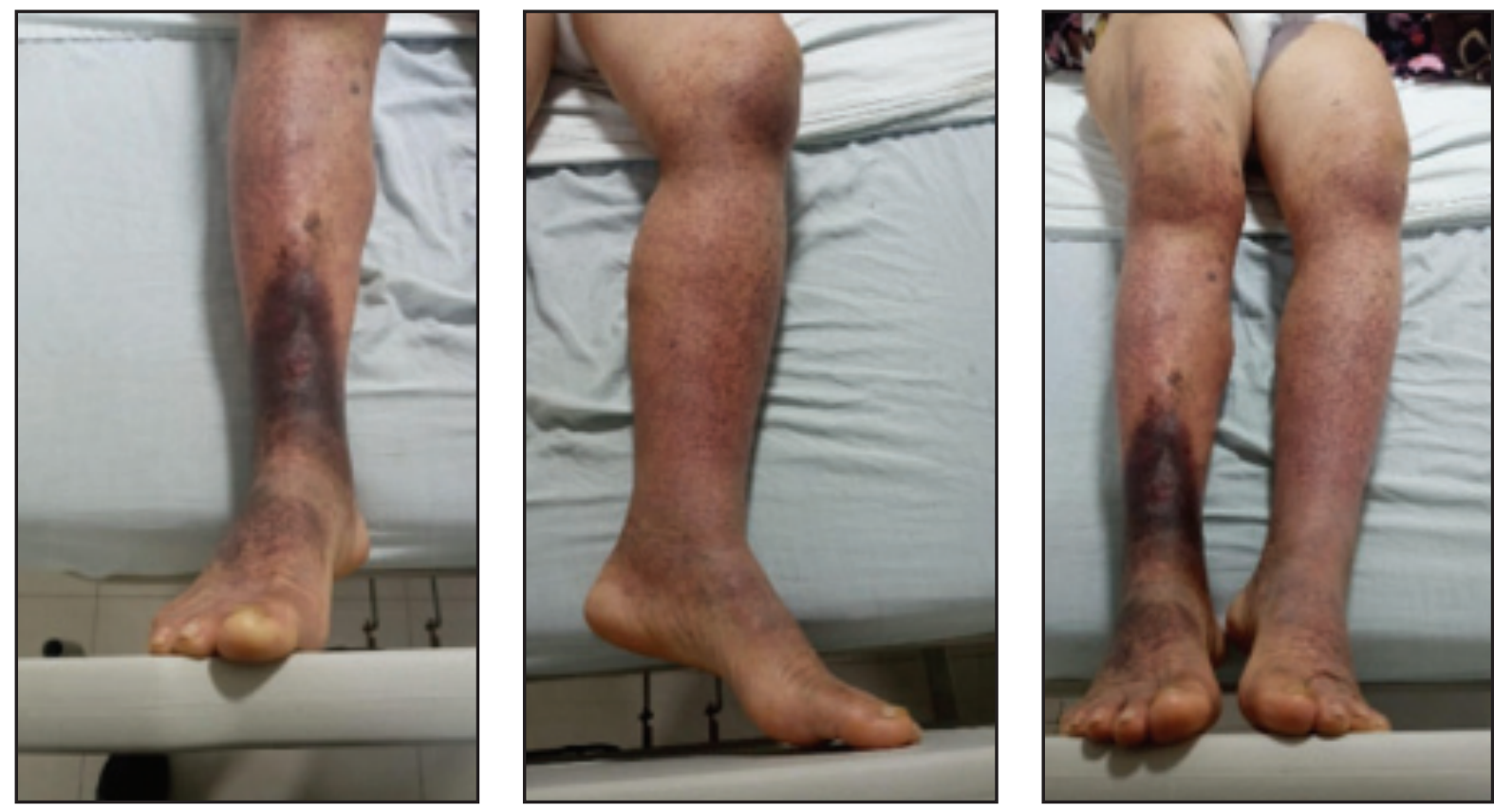

Figuras 1-3. Lesiones purpúricas en miembros inferiores. 
ha demostrado un mecanismo similar a la trombocitopenia inducida por heparina en cuatro pacientes en los que se encontró que los anticuerpos causan una activación plaquetaria a través del receptor Fc (3). El artículo de Schultz et al. (4) describió a cinco trabajadores de la salud de 32-54 años que presentaron tromboembolismo venoso en sitios inusuales y trombocitopenia que ocurrió 7-10 días tras la vacunación. Los pacientes tenían niveles elevados de dímero $\mathrm{D}$ y niveles altos de anticuerpos IgG contra complejos factor plaquetario 4 (PF4) medidos por ELISA. Se inició tratamiento con inmunoglobulina y prednisolona produjeron un aumento del recuento plaquetario. En el trabajo de Greinacher et al., (5) 11 pacientes, de 22-49 años, tuvieron una o más complicaciones trombóticas 5-16 días después de la vacunación. Todos los pacientes tenían trombocitopenia concurrente con un recuento de plaquetas que variaba de 9000-107 000. La prueba serológica de todos los pacientes fue positiva para anticuerpos contra factor plaquetario 4 .

Con respecto al mecanismo por el cual la vacuna desencadena la trombocitopenia inmune, la hipótesis principal se relaciona con la reacción entre el factor plaquetario 4 catiónico y el ADN libre aniónico. Un estudio de 2013 demostró en un modelo murino que el ADN y el ARN forman complejos multimoleculares con PF4 y exponen el epítopo al que se unen los anticuerpos antifactor plaquetario 4 induciendo una respuesta inmune similar a la trombocitopenia por heparina (6).

Varias sociedades médicas han publicado guías sobre cómo abordar dicha reacción adversa (7). Se proponen los siguientes criterios para diagnosticar la trombocitopenia tras la vacunación por COVID-19: aparición de síntomas dentro entre 4-28 días posteriores a la vacunación, trombocitopenia y elevación del dímero D. Todos los pacientes que presenten signos y síntomas que sugieran trombosis o trombocitopenia 4-28 días después de la vacunación contra COVID-19 deben solicitarle un hemograma con estudios de coagulación, los niveles de fibrinógeno están reducidos o inclusive normales (8). La combinación de trombocitopenia y dímero D elevados puede considerarse un criterio suficiente de trombocitopenia inmunológica tras la vacunación. Las pruebas de activación plaquetaria ampliamente disponibles incluyen la liberación de serotonina plaquetaria y el ensayo clásico de activación plaquetaria inducida por heparina (9).

En relación con el manejo anticoagulante, se deben usar anticoagulantes distintos de la heparina, que incluyen inhibidores directos del factor $\mathrm{Xa}$, inhibidores directos de trombina y fondaparinux. Sin embargo, los efectos de administrar heparina a pacientes con dicho evento adverso aún no están claros, con algunas pruebas que sugieren que la heparina podría inhibir la activación plaquetaria mediada por anticuerpos específicamente tras la vacunación para COVID-19. En los pacientes que recibieron inmunoglobulina endovenosa y prednisolona, el recuento de plaquetas aumentó a pesar de continuar el tratamiento con heparina (6). De manera similar, se han analizado muestras de suero de pacientes con trombocitopenia tras la vacunación y se encontró que la activación plaquetaria era inhibida por las heparinas.

Un equipo de la Universidad de Birmingham publicó los resultados de su estudio que investigó los efectos del suero de pacientes con trombocitopenia inducida tras la vacunación sobre la agregación de las plaquetas y evaluó la utilidad de los fármacos disponibles para prevenir su activación. Los autores encontraron que concentraciones bajas $(0.1$ y $0.3 \mathrm{U} / \mathrm{mL})$ y altas $(100 \mathrm{U} / \mathrm{mL})$ de heparina bloquearon la agregación plaquetaria in vitro en muestras de suero obtenidas de pacientes con trombocitopenia inducida tras la vacunación contra el COVID-19 (10). Sin embargo, se necesitan estudios para evaluar el papel de la heparina en la trombocitopenia inducida tras la vacunación, ya que los anticoagulantes distintos de la heparina conllevan un mayor riesgo de hemorragia (11).

Es importante destacar que la transfusión de plaquetas está contraindicada a menos que se someta a un procedimiento invasivo con alto riesgo de hemorragia (12). El cribado generalmente se realiza mediante ELISA para trombocitopenia inducida por heparinas ya que los mecanismos inmunológicos son muy similares y la confirmación requiere tanto ELISA como pruebas de activación.

La presencia de trombocitopenia inducida tras la vacunación para COVID-19 todavía es posible si la prueba ELISA es positivo pero las pruebas de activación plaquetaria arrojan un resultado negativo.

Un equipo de la Universidad de Greifswald desarrolló y evaluó el uso de un ensayo rápido para diagnosticar trombocitopenia inducida tras la vacunación por COVID-19, el ensayo conocido como PIFPA (activación plaquetaria basada en citometría de flujo inducida por PF4) es un ensayo citométrico que se basa en un ensayo de plaquetas lavadas, la prueba de activación plaquetaria inducida por factor plaquetario 4 , tiene una alta especificidad para los anticuerpos relacionados con la vacuna ASTRAZENECA pero por el momento solo se encuentra en laboratorios especializados (13).

Tras la administración de millones de dosis de diferentes tipos de vacunas contra COVID-19 después de la aprobación de emergencia, el desarrollo de eventos adversos graves se monitorea cuidadosamente a nivel mundial. Sin embargo, la reciente aparición de un síndrome con características similares a la trombocitopenia inducida por heparinas después de la vacunación ha provocado algunas alarmas con varias sociedades médicas que brindan recomendaciones sobre el diagnóstico y el tratamiento de este síndrome conocido como síndrome de trombocitopenia tras la vacunación para COVID-19.

Teniendo en cuenta que el riesgo de este tipo de evento sigue siendo extremadamente bajo y los beneficios de la vacunación superan a los riesgos, se necesitan estudios para aclarar la fisiopatología detrás de la trombocitopenia inducida por la vacuna específicamente para COVID-19 y crear estrategias que permitan disminuir el riesgo de eventos adversos. 
Actualmente, el ensayo ELISA factor plaquetario 4-heparina sigue siendo la mejor herramienta de detección, mientras que otras tienen una sensibilidad y utilidad clínica limitadas.

La trombocitopenia inmunológica tras la vacunación para COVID-19 es un área en constante investigación por el momento debemos basarnos en las recomendaciones de las sociedades internacionales sobre el diagnóstico y manejo, por el momento las pruebas moleculares disponibles son limitadas en nuestro entorno, se requiere de una participación multidisciplinaria en este tipo de casos. Las limitaciones de este caso es el no haber podido realizar estudios funcionales y moleculares en específico sobre esta paciente, las preguntas por resolver hasta el momento es determinar cuáles son las estrategias terapéuticas con mejores resultados ya que se carecen de estudios con muestras representativas, la evidencia científica disponible es de baja calidad.

El manejo realizado con corticoides y uso de inmunoglobulina permitió una recuperación del recuento plaquetario tras tres sesiones de esta última. Se continuó un esquema con prednisona ajustado a $1 \mathrm{mg}$ por kilo de peso y seguimiento por hematología.

\section{Referencias}

1. Leng A, Maitland E, Wang S, Nicholas S, Liu R, Wang. Individual preferences for COVID-19 vaccination in China. J. Vaccine. 2021 Jan 8;39(2):247-254. doi: 10.1016/j.vaccine.2020.12.009.

2. Elalamy I, Gerotziafas G, Alamowitch S, Laroche JP, Van Dreden P, Ageno W, Beyer-Westendorf J, Cohen AT, Jimenez D, Brenner B, Middeldorp S, Cacoub P; Scientific Reviewer Committee. Thromb Haemost. 2021 Aug;121(8):982-991. doi: 10.1055/a-1499-0119.
3. Oldenburg, J, Klamroth, R, Langer, F, et al. Diagnosis and management of vaccine-related thrombosis following AstraZeneca COVID-19 vaccination: guidance statement from the GTH. Hamostaseologie. Epub ahead of print 1 April 2021. DOI: 10.1055/a-1469-7481.).

4. Schultz, NH, Sørvoll, IH, Michelsen, AE, et al. Thrombosis and thrombocytopenia after ChAdOx $1 \mathrm{nCoV}-19$ vaccination. $N$ Engl J Med. Epub ahead of print 9 April 2021. DOI: 10.1056/NEJMoa2104882.

5. Greinacher, A, Thiele, T, Warkentin, TE, et al. Thrombotic thrombocytopenia after ChAdOx1 nCov-19 vaccination. $N$ Engl J Med. Epub ahead of print 9 April 2021. DOI: 10.1056/NEJMoa2104840.

6. Jaax, ME, Krauel, K, Marschall, T, et al. Complex formation with nucleic acids and aptamers alters the antigenic properties of platelet factor 4. Blood 2013; 122(2): 272-281.

7. The Thrombosis and Haemostasis society of Australia and New Zealand. Suspected vaccine induced prothrombotic immune thrombocytopenia (VIPIT): THANZ advisory statement for hematologists, https://www.thanz.org.au/documents/item/577.

8. British Society for Hematology. Guidance produced from the Expert Hematology Panel (EHP) focused on Covid-19 Vaccine induced Thrombosis and Thrombocytopenia (VITT), https://b-s-h.org.uk/media/19530/guidance-version-13-on-mngmtof-thrombosis-with-thrombocytopenia-occurring-after-c-19-vaccine_20210407. pdf).

9. Warkentin, TE, Arnold, DM, Nazi, I, et al. The platelet serotonin-release assay. Am J Hematol 2015; 90(6): 564-572.

10. Smith, CW, Kardeby, C, Di, Y, et al. Platelet activation by Vaccine-Induced Immune Thrombotic Thrombocytopenia (VITT) patient serum is blocked by COX, P2Y and kinase inhibitors. medRxiv. 2021. DOI: 2021.2004.2024.21255655.

11. Cuker, A, Arepally, GM, Chong, BH, et al. American Society of Hematology 2018 guidelines for management of venous thromboembolism: heparin-induced thrombocytopenia. Blood Adv 2018; 2(22): 3360-3392

12. Neuro Anesthesia \& Critical Care Society (NACC). Intensive Care guidance for the management of vaccine-associated thrombocytopenia and thrombosis (VATT), https://www.ics.ac.uk/ICS/Pdfs/Standards_Guidelines/Guidance_for_the_management_of_VATT).)

13. Handtke, S, Wolff, M, Zaninetti, C, et al. A Flow cytometric assay to detect platelet activating antibodies in VITT after ChAdOx1 nCov-19 vaccination. Blood. Epub ahead of print 4 May 2021. DOI: 10.1182/blood.2021012064. 